\title{
Are ictal vocalisations related to the lateralisation of frontal lobe epilepsy?
}

\author{
J Janszky, A Fogarasi, H Jokeit, A Ebner
}

\begin{abstract}
The purpose was to analyse whether nonspeech vocalisations in seizures originating in the frontal lobe do have lateralising value. Patients were included who had undergone presurgical evaluation with ictal video-EEG monitoring at the Epilepsy Centre, had had resective epilepsy surgery involving the frontal lobe, and who had remained seizure free $>1$ year postoperatively. Twenty seven patients aged 1-42 years (mean 18) met the inclusion criteria. Age at epilepsy onset ranged from 1 month to 41 years (mean 7.1 years). All selected patients had a unilateral MRI detected lesion within the frontal lobe. Fifteen patients had right sided, 12 patients had left sided epileptogenic zones. Seizures recorded during EEG-video monitoring were re-evaluated to identify the occurrence of ictal vocalisations. Pure ictal vocalisations were distinguished from ictal sound productions due to motor or vegetative seizure activity (for example, cloni or respiratory sounds). Pure ictal vocalisation occurred in 11 patients of whom nine had a left frontal epileptogenic zone $(p<0.01)$. It is concluded that ictal vocalisation could be an additional lateralising sign in frontal lobe epilepsy. The results suggest that not only speech, but vocalisation at a subverbal level also shows a left hemispheric dominance in humans.

(F Neurol Neurosurg Psychiatry 2000;69:244-247)
\end{abstract}

Keywords: vocalisation; frontal lobe epilepsy; hemispheric lateralisation

Ictal speech is a well accepted lateralising sign in temporal lobe epilepsy (TLE). ${ }^{1}$ However, ictal speech is rare in frontal lobe seizures, although non-speech vocalisations often occur. Ictal vocalisations are characteristics of $43 \%$ $63 \%$ of patients having frontal lobe epilepsy (FLE). ${ }^{23}$ Whether non-speech vocalisations are related to the side of seizure origin in FLE is not known. Therefore, we aimed at observing ictal, non-speech vocalisation in patients with FLE in relation to the focus lateralisation. A methodological problem in this field is the fact that sounds of different origin and secondary to ictal motor or vegetative activity may accom- pany seizures (for example, cloni, respiratory sounds). Only audio and video documented seizures may provide sufficient information as to whether speech, vocalisations, or physical sounds are present during a seizure. For the localisation of an epileptogenic zone, the highest amount of certainty can be obtained if a patient becomes seizure free after resective epilepsy surgery. Therefore, we exclusively reevaluated audio and video documented seizures in patients who had become seizure free after frontal lobe surgery.

\section{Methods}

All patients were included in this study who had consecutively undergone a presurgical evaluation between 1992 and July 1998 at our institution, had had resective epilepsy surgery involving the frontal lobe, and who had remained seizure free for more than 1 year postoperatively.

Twenty seven patients, 20 men and seven women, met the inclusion criteria. Age at video-EEG monitoring was $1-42$ (mean 18) years. Age at epilepsy onset ranged from 1 month to 41 (mean 7.1) years. The postsurgical follow up ranged between 1 and 5 years. All the patients had MRI and long term monitoring with interictal and ictal scalp EEG, and ictal audio-video recordings. In 17 patients, the epileptogenic zone was delineated by using chronic subdural electrodes. To determine the speech dominance, a Wada test was performed in 12 patients. Ten patients had left and two had right hemispheric speech dominance. In the remaining 15 patients, the Wada test was not performed due to their age (five patients were younger than 5) or because the localisation of the epileptogenic area was far from the speech areas (frontomesial and orbitopolar focus localisation). Fifteen patients had right sided and 12 patients had left sided epileptogenic zones. All selected patients had an lesion in the frontal lobe detected by MRI. Histopathological examination disclosed the following abnormalities: focal cortical dysplasia 17 patients; tumour six patients; arteriovenous malformation one patient; ischaemic lesion three patients. The most common resection was an extended lesionectomy; in some cases, especially in orbitopolar regions, a more extended surgery was perfomed on the basis of epileptological data. Postoperative MRI con- 
Ictal speech and non-speech sound production in patients with right and left sided epileptogenic foci

\begin{tabular}{llllll}
\hline & $\begin{array}{l}\text { All patients } \\
(n=27)\end{array}$ & $\begin{array}{l}\text { Right focus } \\
(n=15)\end{array}$ & $\begin{array}{l}\text { Left focus } \\
(n=12)\end{array}$ & p Value & $\kappa$ Value \\
\hline $\begin{array}{l}\text { Ictal speech } \\
\begin{array}{l}\text { Ictal, non-speech sound } \\
\text { productions }\end{array}\end{array}$ & 21 & $0(0 \%)$ & $2(17 \%)$ & 0.18 & 1.0 \\
$\begin{array}{l}\text { Sounds of apnoea, GTCS, or } \\
\text { clonus }\end{array}$ & 14 & $9(60 \%)$ & $12(100 \%)$ & 0.02 & 0.81 \\
$\begin{array}{l}\text { Pure ictal vocalisation } \\
\begin{array}{l}\text { Postictal vocalisation } \\
\text { Number of seizures (mean }\end{array}\end{array}$ & 11 & $9(60 \%)$ & $5(42 \%)$ & 0.34 & 0.63 \\
$\quad 2.7 \pm 2$ & $2(13 \%)$ & $9(75 \%)$ & 0.002 & 0.92 \\
$\quad(S D))$ & $5.8(2.4)$ & $5.6(1.5)$ & 0.79 & 0.78 \\
\end{tabular}

GTCS $=$ Generalised tonic-clonic seizures.

firmed a complete removal of the lesions in all patients.

All ictal audio-video recordings of each patient were selected for evaluation except if the patient had more than nine recorded seizures. In this case we selected only the first nine consecutive recordings. Seizures stored on videotape were reviewed by two of us (JJ and $\mathrm{AF}$ ), one of us (AF) was blinded to the patients' clinical data. Except for the measurement of interobserver agreement, only the findings of the blinded investigator were used. Audible sounds of ictal audio-video recordings were divided into the following categories:

- Intelligible speech

- Ictal, non-speech sounds

- Sounds accompanying apnoea, clonic, or generalised tonic-clonic seizures (GTCS)

- Pure ictal vocalisation (PIV)

- Postictal vocalisation.

An audible sound was defined as PIV if the vocalisation produced by the patient had no speech quality, did not accompany apnoea, GTCS, or clonic seizure, and was obviously an ictal phenomenon. We registered the character of PIV, its association with affective changes or intelligible/unintelligible speech, accompanying seizure type, and whether the PIV was the first clinical seizure sign. The PIV phenomenon was considered to be present in a patient if it occurred during at least one of the evaluated seizures. For analysis of categorical data, $\chi^{2}$ and Fisher's exact tests were performed. For continuous variables, the MannWhitney test was used. Two tailed error probabilities $<0.05$ were considered to be significant. The interobserver agreement was determined by the $\kappa$ coefficient.

\section{Results}

We analysed 153 seizures of 27 patients (mean 5.7 per patient). Tonic seizures were present in $67 \%$, complex partial seizures (CPS) in $48 \%$, clonic seizures in $18 \%$, and myoclonic seizures in $11 \%$ of the patients. In $37 \%$ of all patients, CPS were characterised by motor agitation ("hypermotor seizures"). ${ }^{4}$ In $41 \%$ of all cases, tonic seizures involved only one limb. Thirteen patients had more than one seizure type. The table shows the characteristics of the sound productions. Pure ictal vocalisation occurred in 11 patients, in six of them during CPS; in seven during tonic seizure (two patients had PIV during both seizure types). PIV was the first seizure sign in six patients. In two patients, it was accompanied by intelligible, in four by unintelligible speech. Six patients showed negative emotional components during PIV. In nine patients, PIV had a repetitive character. Groaning or grumbling occurred in eight, shouting in five, and weeping in one patient. In
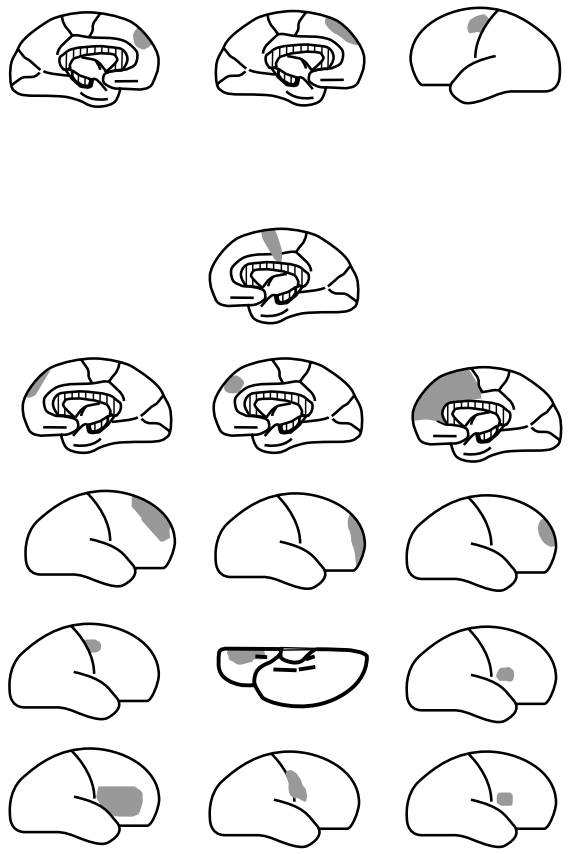

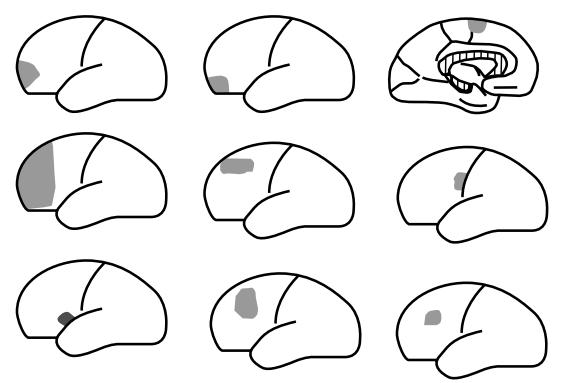

Epileptogenic lesions of patients with (on the right side of the figure) or without (on the left side of the figure) pure ictal vocalisation (PIV). Patients with PIV more often had left hemispheric lesions $(p=0.002)$ and dorsolateral localisation; however, the latter was not significant 
all variables, the degree of interobserver agreement was sufficient ( $\kappa$ ranged between 0.6 and 1.0).

Ictal, non-speech sounds occurred significantly more often in patients with a left sided epileptogenic zone than with right sided epileptogenic focus (table). All the patients who had no clear audible sounds during video recordings had right sided foci. Moreover, observing only the PIV, this difference was highly significant. Ictal speech, sounds due to vegetative or motor seizure activity, postictal vocalisations, and the total number of seizures showed no significant difference in patients with left versus right focus. There was a trend towards the dorsolateral localisation of the epileptogenic lesion in patients with PIV. Seven patients with PIV had dorsolateral (64\%), one fontomesial (9\%), and three orbitopolar $(27 \%)$ lesions. Six patients without PIV had dorsolateral $(37.5 \%)$, six frontomesial $(37.5 \%)$, and four orbitopolar $(25 \%)$ epileptogenic zones (figure).

As the Wada test was performed in only 12 patients, we were not able to correctly investigate PIV in relation to hemispheric speech dominance. Of the two patients with right sided speech dominance, one had a left focus and had a PIV during the seizure, the other had a right focus and no PIV.

\section{Discussion}

We found that epileptic patients with left sided frontal foci had significantly more frequent ictal, non-speech vocalisations than patients with right sided frontal foci.

Only one study mentioned lateralisation differences of ictal vocalisation in frontal lobe epilepsy (FLE). Studying the data of 210 patients with FLE, Bancaud and Talairach stated that the vocalisation was characteristic of the seizures originating from the dominant inferior frontal gyrus. ${ }^{6}$ However, this finding was mentioned only in a table, without defining the relative frequency or lateralisation value of ictal vocalisation. Moreover, these data were based on personal findings without blinded investigators. In this study, vocalisation was also a prominent feature of seizures originating from the supplementary motor area and anterior cingulate gyrus; however, lateralisation differences were not reported. Another study dealing with 16 patients with $\mathrm{FLE},{ }^{3}$ found no association between the lateralisation and vocalisation; however, no distinction was mentioned between PIV and sounds due to motor or vegetative ictal activity. Gabr et al found that patients with TLE with dominant foci had ictal vocalisations more often than patients with non-dominant foci $(62 \% v 37 \%)$, but this trend was not significant. ${ }^{1}$

There are some physiological arguments for the lateralisation of vocalisation. Vocalisation could be elicited by direct electrical stimulation of two frontal lobe areas. Stimulating the Broca area, the common response is an interference with language production ${ }^{7}$; however, in some cases, a vocalisation could also be evoked from the Broca area and also from the contralateral Broca homologue area. ${ }^{8}$ When stimulating the supplementary motor area, vocalisation appeared more often in the dominant than in the non-dominant supplementary motor area. ${ }^{9} 10$ The presence of aphasic symptoms after the infarction or removal of the left supplementary motor area also supports a lateralised participation of the region in sound generation. ${ }^{11} 12$ During automatic speech, the dominant supplementary motor area shows a more marked hyperperfusion than the non-dominant area. ${ }^{13}$ Moreover, the lateralisation of sound production is not a specific human phenomenon; subhuman vertebrates seem to have left hemispheric dominance of vocal productions. ${ }^{14}$

We are unable to explain the mechanism of the lateralisation of ictal vocalisation. The relation between clinical manifestations and the precise site of seizure origin is generally problematic in FLE because of the rapid propagation into the adjacent or distant cortex, or even to the contralateral frontal lobe. ${ }^{15}$ Consequently, many ictal manifestations may result from seizure spread. ${ }^{3}$ With this limitation in mind, we may suspect two areas, the Broca and the supplementary motor areas, in the generation of PIV. These areas show lateralised and non-lateralised features in the generation of sound production. The trend towards the dorsolateral localisation of the epileptogenic focus in our patients with PIV and the findings of Bancaud and Talairach may raise the possibility that the lateralisation of PIV in FLE may be primary caused by the activation of the Broca region.

Although the distinction between the PIV and sounds due to motor and vegetative seizure activity may have subjective elements, we found a sufficient interobserver agreement. This distinction, difficult to express semantically, is comparable with that between dystonic versus tonic posturing, head version versus head deviation, ${ }^{16}$ cloni versus "shaking" in pseudoseizures, and unilateral blinking versus facial cloni. ${ }^{17}$

Tonic seizure was the most common seizure type in our patients. Some authors hypothesised that vocalisation in tonic seizures is a result of involuntary ictal contraction of diaphragmatic and laryngeal muscle. ${ }^{18}$ This assumption has not been confirmed. In seven patients with tonic seizures, we evaluated the ictal sound production as PIV, as an apnoeic event was not obvious. Most of these seizures involved only one limb, without "generalised" features. The fact that PIV in most patients had a repetitive character, also supports the view that PIV in tonic seizures is not a consequence of a tonic pharyngeal contraction.

One of the limitations of our study is that we had data about the speech lateralisation in only 12 patients; consequently we could not investigate whether PIV relates on the right-left lateralisation or on the speech dominance. Another limitation is that the number of patients is small. Furthermore, the selection of patients was retrospective, and we evaluated only patients with circumscribed epileptogenic area, and who remained postoperatively seizure free, which might also cause a selection bias. Due to 
these limitations, the diagnostic value of PIV has to be evaluated in prospective studies.

In conclusion, we found that ictal vocalisation could be a lateralisation sign in frontal lobe seizures. The frequent presence of PIV in patients with left sided FLE $(75 \%)$ and its rare occurrence in patients with right sided FLE $(13 \%)$ may improve the probability of a correct lateralisation of the epileptogenic zone during the diagnostic process. The lateralisation of the PIV suggests that not only speech but the vocalisation at a subverbal level also shows a left hemispheric dominance in humans.

This work was supported by a grant from the Deutsche Forschungsgemeinschaft (DFG-Eb 111/2-2) and from the Society for Epilepsy Research Bethel (JJ and AF).

1 Gabr M, Lüders H, Dinner D, et al. Speech manifestations in lateralization of temporal lobe seizures. Ann Neurol 1989;25:82-7.

2 Ferrier CH, Engelsman J, Alarcón G, et al. Prognostic factors in presurgical assessment of frontal lobe epilepsy. $\mathcal{F}$ Neurol Neurosurg Psychiatry 1999;66:350-6.

3 Laskowitz DT, Sperling MR, French JA, et al. The syndrome of frontal lobe epilepsy: characteristics and surgical management. Neurology 1995;45:780-7.

4 Manford M, Fish DR, Shorvon SD. An analysis of clinical seizure patterns and their localizing value in frontal and temporal lobe epilepsies. Brain 1996;119:17-40.
5 Lüders HO, Acharya J, Baumgartner C, et al. Semiological seizure classification. Epilepsia 1998;39:1006-13.

6 Bancaud J, Talairach J. Clinical semiology of frontal lobe seizures. Adv Neurol 1992;57:3-37.

7 Lüders HO, Lesser RP, Dinner DS, et al. A negative motor response elicited by electrical stimulation of the human frontal cortex. Adv Neurol 1992;57:361-8.

8 Penfield W, Rasmussen T. Vocalization and arrest of speech. Arch Neurol Psychiatry 1949;61:21-7.

9 Fried I, Katz A, McCarthy G, et al. Functional organization of human supplementary motor cortex studied by electrical of human supplementary motor cortex stu

10 Chauvel P, Bancaud J, Buser P. Participation of the supplementary motor area in speech. Exp Brain Res 1985;58:A14.

11 Funkenstein H, Masdeu JC, Schoene WC. Aphasia following infarction of the left supplementary area: a clinicopathological study. Neurology 1978;28:1220-3.

12 Rostomily RC, Berger MS, Ojemann GA, et al. Postoperative deficits and functional recovery following removal of tumors involving the dominant hemisphere supplementary motor area. F Neurosurg 1991;75:62-8.

13 Larsen B, Skinhøj E, Lassen N. Variations in regional cortical blood flow in the right and left hemispheres during automatic speech. Brain 1978;101:193-209.

14 Walker SF. Lateralization of functions in the vertebrate brain: a review. Br 7 Psychol 1980;71:329-67.

15 Ebner A, Buschsieweke U, Tuxhorn I, et al. Supplementary sensorimotor area seizures and ictal single-photon emission tomography. Adv Neurol 1996;70:363-8.

16 Jayakar P, Duchowny M, Resnick T, et al. Ictal head deviation: lateralizing significance of the pattern of head movement. Neurology 1992;42:1989-92.

17 Benbadis SR, Kotagal P, Klem GH. Unilateral blinking: a lateralizing sign in partial seizures. Neurology 1996;46:458.

18 Bleasel AF, Morris HH. Supplementary sensimotor area epilepsy in adults. Adv Neurol 1996;70:271-9. 